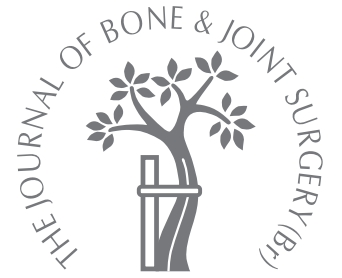

F. Langlais, N. Belot, M. Ropars, J. C. Lambotte, H. Thomazeau

From the University Hospital, Rennes, France

\title{
The long-term results of press-fit cemented stems in total knee prostheses
}

We evaluated the long-term fixation of 64 press-fit cemented stems of constrained total knee prostheses in 32 young patients with primary malignant bone tumours. Initial stable fixation, especially in rotation, was achieved by precise fit of the stem into the reamed endosteum, before cementation. Complementary fixation, especially in migration and rotation, was obtained by pressurised antibiotic-loaded cement. The mean age at operation was 33 years (13 to 61). No patient was lost to follow-up; 13 patients died and the 19 survivors were examined at a mean follow-up of 12.5 years (4 to 21). Standard revision press-fit cemented stems were used on the side of the joint which was not involved with tumour (26 tibial and six femoral), on this side there was no loosening or osteolysis and stem survival was $100 \%$. On the reconstruction side, custom-made press-fit stems were used and the survival rate, with any cause for revision as an end point, was $88 \%$, but $97 \%$ for loosening or osteolysis. This longevity is similar to that achieved at 20 years with the Charnley-Kerboull primary total hip replacement with press-fit cemented femoral components.

We recommend this type of fixation when extensive reconstruction of the knee is required. It may also be suitable for older patients requiring revision of a total knee replacement or in difficult situations such as severe deformity and complex articular fractures.

When knee prostheses are revised for loosening or osteolysis, satisfactory results are achieved usually by the reconstruction of the epiphyseal region and the use of alignment stems, which are not fixed into the diaphysis in order to avoid stress shielding. However, diaphyseal fixation is sometimes necessary in the presence of major bone loss and when prostheses are used following resection of tumours. In the latter case, diaphyseal fixation is also needed on the side of the joint which is not involved by the tumour. In these circumstances the stems may be porouscoated, or have a bioactive coating, or can be cemented. Fixation of the cemented stem can be achieved either by using an even mantle of cement or by using a press-fit component.

In total hip replacement, diaphyseal press-fit cemented stems have shown a long-term survival of over $95 \%$ at 20 years' follow-up. ${ }^{1}$ We describe a consecutive series of reconstruction total knee prostheses using press-fit cementation.

\section{Patients and Methods}

This consecutive series comprised of 32 total knee prostheses with press-fit stems which were used in patients with primary malignant tumours between 1982 and 2000. During this period we also used other types of reconstruction prostheses, particularly Link rotating hinges (Link GmbH, Hamburg, Germany) with an even mantle of cement and a centraliser. The mean age of the patients was 33 years (13 to 61). There were 17 females and 15 males.

There were 18 osteosarcomas, nine other high malignant sarcomas, such as Ewings and malignant histiocytofibromas, and five chondrosarcomas. Neo-adjuvant chemotherapy was used in 27 patients, associated with post-operative radiotherapy in eight. No patient was lost to follow-up, but 13 died in the first three years after operation. A hinged prosthesis with press-fit cemented stems was used (Fig. 1). In 16 cases, the articulation of the tibial and the femoral components was by bushings, which were of polyethylene in eight cases and of metal-on-metal (cobalt-chrome) in eight. Wear of these bushings caused synovitis of the knee, so a different design has been used in 16 cases since 1993. This articulation has metal-onmetal bearings of cobalt-chrome with optimised clearance and roughness, and a double mobility of the axle. The patella was resur- 

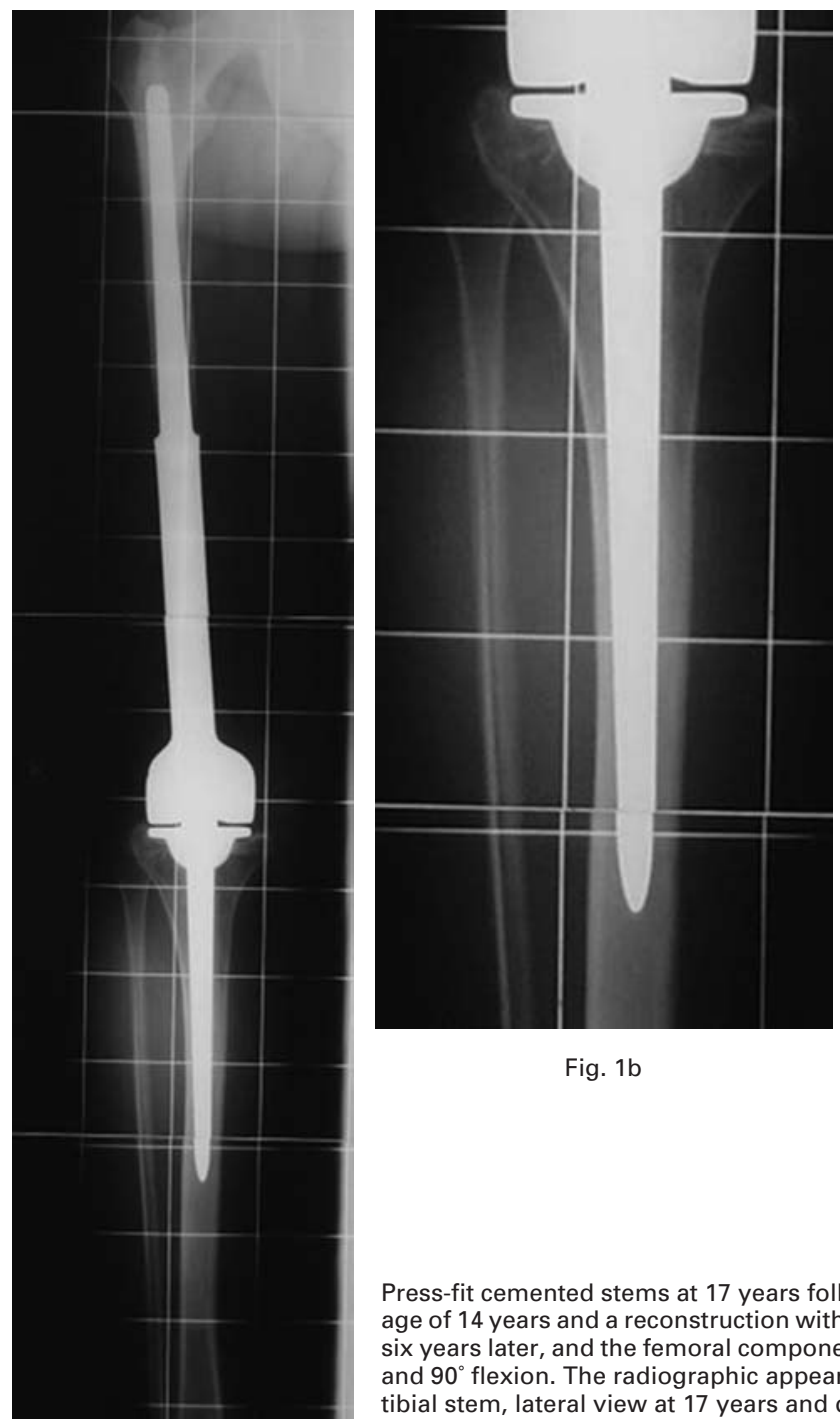

Fig. 1b

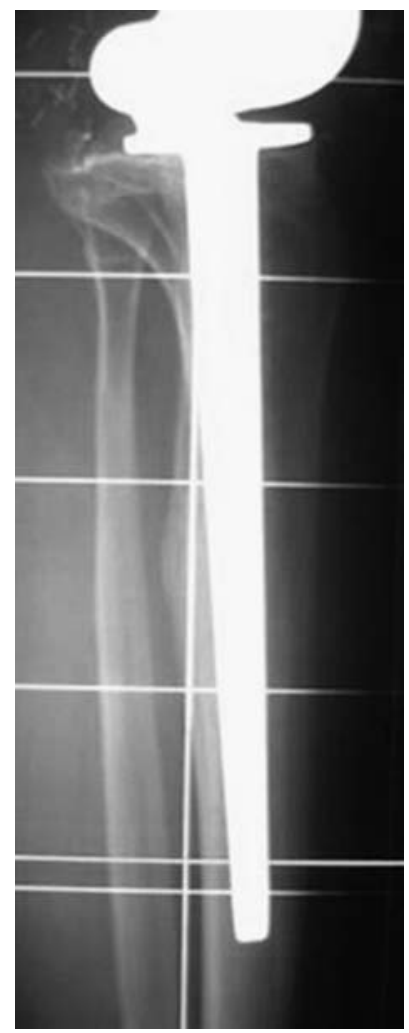

Fig. 1c

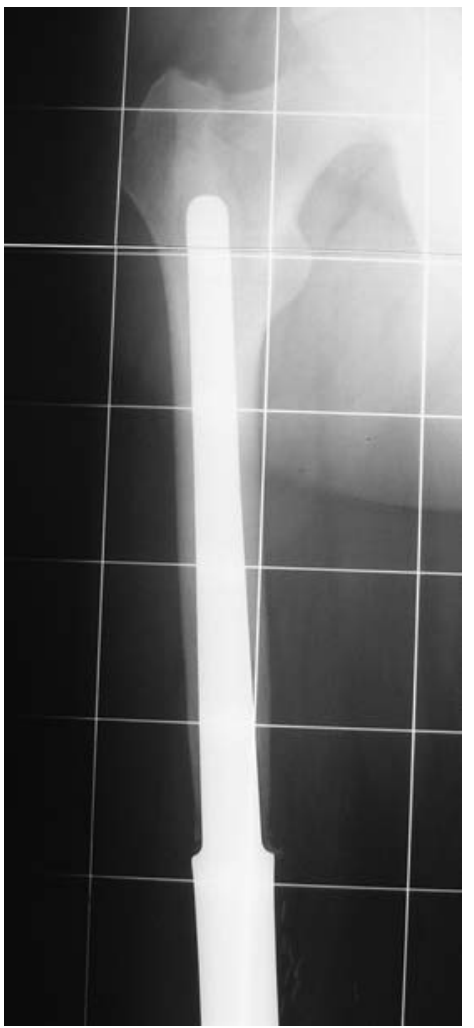

Fig. 1d

Press-fit cemented stems at 17 years follow-up. This patient had a distal femoral resection for an osteosarcoma at the age of 14 years and a reconstruction with a press-fit cemented stem prosthesis. A fracture of the femoral stem occurred six years later, and the femoral component was exchanged. At 31 years of age, he has good function with an active life and $90^{\circ}$ flexion. The radiographic appearances show a) the global view; b) the tibial stem, anteroposterior view; c) the tibial stem, lateral view at 17 years and d) the proximal femur (at 11 years after exchange).

Fig. 1a

faced with a dome-shaped prosthesis in 16 cases. The extensor apparatus was mobilised in 11 cases to achieve a wide resection; reconstruction in five was by transfer of hamstring muscles ${ }^{2}$ and in six the patellar ligament was replaced either by a fibular flap ${ }^{3}$ or with a tibial bonetendon combined composite prosthesis. ${ }^{4}$

On the side of the joint not involved by tumour a standard revision stem was used (Guepar II, Stryker France, Lyon, France). There were 26 femoral and six tibial tumours and thus 26 standard tibial and six standard femoral revision stems were used. The revision stems were characterised by their quadrangular shape (Fig. 2) with sharp edges biting into the diaphyseal endosteum. The stem was $180 \mathrm{~mm}$ long and tapered. Preparation was achieved by broaches at the metaphysis and by progressive reaming of the diaphysis. Reaming increased progressively by 0.5 $\mathrm{mm}$ increments until the trial prosthesis could be stabilised without cement in the sagittal and coronal planes and in rotation. Once this primary stability had been achieved by the geometric fit of the prosthesis, second generation cementing was used with plugging of the canal, pulsed lavage and retrograde injection of gentamicin-loaded cement using a syringe and strong pressurisation.

Custom-made stems were used on the side of the tumour. The $170 \mathrm{~mm}$ long stem had two parts (Fig. 1). The cylinder was $50 \mathrm{~mm}$ long with a diameter $1 \mathrm{~mm}$ to $2 \mathrm{~mm}$ greater than that of the medullary canal, the diameter being $14 \mathrm{~mm}$ in 26 cases. The rest of the stem was tapered down to a diameter of $2 \mathrm{~mm}$ along $100 \mathrm{~mm}$ of length with three slots preventing rotation. The diaphysis was reamed with increments of $0.5 \mathrm{~mm}$, so that the cylindrical part of the stem could fit exactly into the reamed endosteum with good control of rotation. The tight fit of the cylindrical part of the stem resulted in high pressurisation of the cement.

Thus, 64 press-fit cemented stems were used in total, including 32 standard revision stems. The stem had to support extremely high strains due to the activity of these young patients, as well as the non-rotating hinge and exten- 


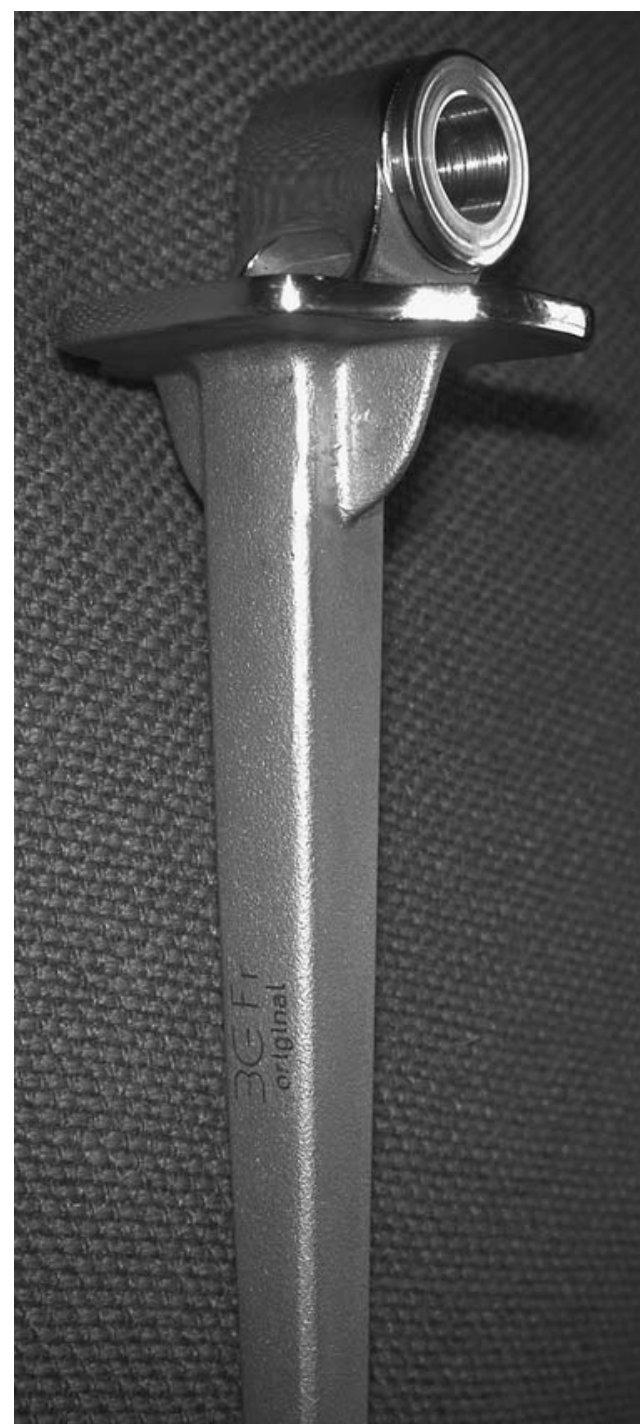

Fig. 2

The tapered quadrangular tibial revision stem.

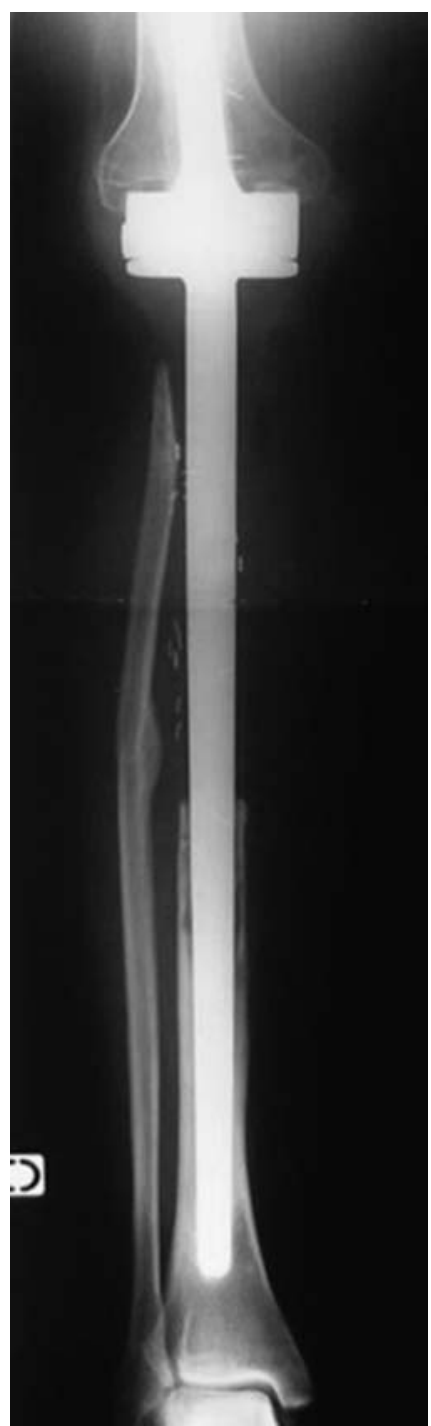

Fig. 3

Osteolysis about the tibial stem at 12 years follow-up. This 13-year-old girl had a proximal tibial osteosarcoma with a reconstruction prosthesis. Because of osteolysis of the proximal tibia, the tibial prosthesis was revised using an allograft composite prosthesis. sive resection of bone and soft tissues. The mean length of bony resection was $185 \mathrm{~mm}$ (100 to 260).

In the 27 patients who had post-operative chemotherapy there was a risk of secondary infection ${ }^{5,6}$ due to leucopenia, a possible limitation of bone remodelling due to chemotherapy and, therefore, weakened fixation.

\section{Results}

Of the 32 patients, 13 died in the first three years, 11 due to pulmonary metastases in tumours with a poor response to the chemotherapy and two due to unrelated causes.

There were no infections and no evidence of mechanical failure of the prostheses in these 13 patients. In the 19 surviving patients, the mean follow-up was 12.5 years (4 to 21).
Fixation of the stem. In the 32 standard revision stems, there was no evidence of loosening, diaphyseal osteolysis or cortical thickening. In the tibial prostheses, $1 \mathrm{~mm}$ or $2 \mathrm{~mm}$ of resorption was seen under the prosthetic tibial plateau during the first post-operative year, but this was not progressive. The stems showed no modification of fixation up to 20 years post-operatively.

Some changes were seen around the stem on the tumour side of the joint. One tibial stem $(3 \%)$ in a girl aged 13 years with an osteosarcoma required revision after 12 years because of endosteal resorption (Fig. 3). Revision with a composite prosthesis and a press-fit cemented stem gave a good result after eight years follow-up. The cause of the osteolysis may have been polyethylene debris coming from 
Table I. Results of total knee reconstruction prostheses at long- and medium-term follow-up

\begin{tabular}{|c|c|c|c|c|c|c|c|c|}
\hline Authors & $\begin{array}{l}\text { Mean follow- } \\
\text { up (yrs) }\end{array}$ & Fixation & $\begin{array}{l}\text { Type of } \\
\text { prosthesis }\end{array}$ & $\begin{array}{l}\text { Type of } \\
\text { hinge }\end{array}$ & $\begin{array}{l}\text { Bearing } \\
\text { surfaces* }\end{array}$ & $\begin{array}{l}\text { Loosening or } \\
\text { lysis revision (\%) }\end{array}$ & $\begin{array}{l}\text { Stem fracture } \\
(\%)\end{array}$ & $\begin{array}{l}\text { Deep } \\
\text { infections (\%) }\end{array}$ \\
\hline Mittermayer et al ${ }^{14}$ & $\geq 10$ & Not cemented & Kotz & Plain & $\mathrm{M} / \mathrm{PE}$ & 27 & 10 & 9 \\
\hline Unwin et $\mathrm{al}^{19}$ & $\geq 10$ & Cemented & Stanmore & Plain & $\mathrm{M} / \mathrm{PE}$ & $\simeq 30$ & - & 8 \\
\hline Bickels et $\mathrm{al}^{21}$ & $\geq 10$ & Cemented & Kinematic & Rotating & $\mathrm{M} / \mathrm{PE}, \mathrm{C}$ & 6 & - & 5 \\
\hline Current series & $\geq 10$ & $\begin{array}{l}\text { Cemented- } \\
\text { press-fit }\end{array}$ & Guepar & Plain & $\mathrm{M} / \mathrm{PE}$ and $\mathrm{M} / \mathrm{M}$ & 3 & 6 & 6 \\
\hline Horowitz et al ${ }^{15}$ & $<5$ & Not cemented & Lane & Plain & $\mathrm{M} / \mathrm{M}$ & 25 & - & - \\
\hline Capanna et al ${ }^{16}$ & $<5$ & Not cemented & Kotz & Plain & $\mathrm{M} / \mathrm{PE}$ & 25 & 6 & - \\
\hline Lindner et $\mathrm{al}^{8}$ & $<5$ & Not cemented & - & - & $\mathrm{M} / \mathrm{PE}$ & $\simeq 20$ & - & - \\
\hline Plötz et al ${ }^{17}$ & $<5$ & Not cemented & - & - & $\mathrm{M} / \mathrm{PE}$ & 11 & 22 & 3 \\
\hline Mascard et $\mathrm{al}^{20}$ & $<5$ & Cemented & $\begin{array}{l}\text { Modified } \\
\text { Guepar }\end{array}$ & $\begin{array}{l}\text { Plain and } \\
\text { rotating }\end{array}$ & $\mathrm{M} / \mathrm{PE}$ & 13 & 5 & 10 \\
\hline Kawai et $\mathrm{al}^{24}$ & $<5$ & Cemented & Finn & Rotating & $\mathrm{M} / \mathrm{PE}, \mathrm{C}$ & - & - & 12 \\
\hline
\end{tabular}

* M/PE, metal and polyethylene; C, large congruity; M/M, metal-on-metal

micromotion of a polyethylene sheath on the stem at the level of resection of the tumour. We did not use this type of sheath in other cases.

There were two fractures $(6 \%)$ of firmly-fixed femoral stems at six and seven years after implantation. These occurred in two young patients (aged 13 and 14 years) who were not skeletally mature at operation and required implantation of thin stems, $12 \mathrm{~mm}$ in diameter. New pressfit cemented stems, $14 \mathrm{~mm}$ in diameter, gave satisfactory results with a follow-up of more than 10 years.

In four cases, there was moderate and asymptomatic resorption of bone. This occurred at $40 \mathrm{~mm}$ from the zone of resection with cortical lysis of between $2 \mathrm{~mm}$ and $6 \mathrm{~mm}$ in depth. These changes were seen after two years and did not progress. Fixation of the stem remained sound. In two of these cases, synovitis of the knee due to debris from wear of the hinge was also seen.

In the 25 other cases there was no radiolucency, osteolysis, cortical thickening or cortical resorbtion.

At maximum follow-up, the global survival of the prosthesis was $88 \%$ (three stems exchanged on 32 prostheses) and the survival for revision for loosening or osteolysis was 97\% (one exchange for osteolysis on 32 prostheses).

Function. The oncological and functional results were similar to those reported in the literature for oncological cases. ${ }^{7-12}$

According to the Musculoskeletal Tumor Society rating ${ }^{13}$ the mean overall function of the survivors was $81 \%$ of normal. All 19 surviving patients had a normal family life with no strenuous hobbies.

Movement. In the 24 patients who did not have radiotherapy, the median flexion of the knee at follow-up was $105^{\circ}$ with a mean of $85^{\circ}\left(60^{\circ}\right.$ to $\left.130^{\circ}\right)$. The eight knees in patients who had radiotherapy had a median flexion of $44^{\circ}$. Satisfactory active extension was achieved in nine of the 11 patients who underwent reconstruction of the extensor apparatus.

Complications. There were some complications which were not related to stem fixation. Post-operative skin necrosis occurred in six patients and in a further six there was a loss of active movement because of excision of the muscles and nerves.
In the eight prostheses with plain hinge and polyethylene bushings, six revisions were necessary for wear of the bushings which had caused laxity and synovitis. Exchange with metal bushings proved disappointing because in half of these, synovitis due to metallosis occurred, requiring further exchange of the bushings and synovectomy. However, in the 16 non-rotating hinges with modified metal-on-metal bearings without bushings, we did not observe any wear with laxity, osteolysis, or loosening, and only minor synovitis in one patient.

Of the 32 patients, 17 (53\%) required a re-operation for reasons not related to progression of the tumour.

No peri-operative infection occurred. There were two secondary infections $(6 \%)$ occurring 20 and 21 years after implantation in prostheses with well-fixed stems but with significant synovitis and metal bushings. One was secondary to a urinary infection and the other to erysipelas. Both were satisfactorily treated by a two-stage exchange procedure.

\section{Discussion}

This series demonstrates the reliability and longevity of the fixaton of press-fit cemented stems. We compared this with other series in the literature in Table I.

The results with uncemented stems are only fair. The first generation of these stems was made of chrome-cobalt bioactive coating and had a non-rotating hinge. They were characterised by considerable bone resorption due to stress shielding, with a loosening rate of about $25 \%$ at ten years. ${ }^{14-16}$ Improvement in design led to better longevity but the rate of loosening at five years remained more than $10 \% .{ }^{17}$ We are not aware of the long-term results of reconstruction prostheses with a fully bioactive coating of hydroxyapatite. ${ }^{18}$

In a series of hinged prostheses whose stems were fixed using an even mantle of cement, a rate of loosening of approximately $30 \%$ over ten years was reported. ${ }^{19}$ Similarly, we observed in our series of Link reconstruction prostheses, which have a rotating hinge with uniform cement mantle achieved by a centraliser, a rate of loosening 
of $27 \%$ at ten years (unpublished data). Other series with variants of the Guepar hinge also showed disappointing results with an approximate loosening rate of $15 \%$ at ten years. ${ }^{20}$ In these series the dimensions of the stems or the principles of fixation were different from ours. In paediatric oncology the prostheses often had thin stems to spare the growth plate and were not press-fit; many had rotating hinges which produced polyethylene wear debris. ${ }^{20}$ In the adult cases, the modified stems on the side of the tumour resection were smaller than ours with lengths of $120 \mathrm{~mm}$, whereas the diameter of their proximal part was also smaller and did not require endosteal reaming. ${ }^{20}$ Primary fixation by precise press-fit before cementation was therefore not achieved, and the mechanical conditions were close to those of stems with an even cement mantle.

Press-fit cemented fixation can be achieved with modular prostheses. This has been shown in a series of 110 reconstruction prostheses with a rotating hinge and press-fit cemented stems which achieved a rate of loosening of only $6 \%$ at ten years. ${ }^{21}$

The results of our series are similar to those observed with total hip prostheses with press-fit cemented stems, according to the principles of the 'French Paradox'. ${ }^{1}$ Which factors can explain the long-term fixation achieved in our series of reconstruction prostheses? On the normal side of the joint using standard revision stems, there may be a distribution of the strain between the bone and the cement due to the direct transmission of forces to the endosteum by the sharp edges of the stem. The reduced strains on the cement avoid fatigue fracture and the cement contact with the flat side of the stem helps control rotation. The tapered diameter acts as a self-locking mechanism.

On the side of the reconstruction, there is also a share of the strains transmitted by the stem between the endosteum and the cement. Moreover, the close fit achieves a major pressurisation of the cement. However, the custom-made stem does not have the same shape as the other stem and may be more exposed to mechanical loosening, especially if the fit achieved at operation is suboptimal. Fixation could be even better with a more physiological articulating system of a rotating hinge $\mathrm{e}^{22-24}$ and with less wear debris with improved designs. Sealing of the stem-endosteal interface by hydroxyapatite rings could avoid penetration of wear debris along the stem and the subsequent osteolysis.

The longevity of the press-fit cemented stem is of particular interest in two circumstances. First, in oncological reconstruction surgery the use of antibiotic-loaded cement helps to prevent post-operative infection and helps the fixation of allografts in composite prostheses of the proximal tibia, when a flap ${ }^{25,26}$ or a transfer ${ }^{4,27}$ cannot be achieved. Secondly, for revision surgery the diaphyseal fixation of a constrained prosthesis may allow early weight-bearing in addition to a lower rate of infection. It may also be recommended as a primary replacement in some specific indications for older patients, such as significant deformities or severe articular fractures.
No benefits in any form have been received or will be received from a commercial party related directly or indirectly to the subject of this article.

\section{References}

1. Langlais F, Kerboull M, Sedel L, Ling RS. The "French paradox". J Bone Joint Surg [Br] 2003;85-B:17-20.

2. Capanna R, Ruggieri P, Biagini R, et al. The effect of quadriceps excision on functional results after distal femoral resection and prosthetic replacement of bone tumors. Clin Orthop 1991;267:186-96.

3. Langlais F, Belot $\mathbf{N}$, Thomazeau $\mathbf{H}$, et al. Tumeurs malignes osseusus du genou: exérèse et reconstruction. Techniques Chirurgicales-Orthopédie-Traumatologie 2005;44-093:1-15.

4. Langlais F, Delepine G, Dubousset JF, Missenard G. Composite prostheses in malignant tumors: rationale and preliminary results of 42 cases. In: Langlais $F$ Tomeno B, eds. Limb salvage: major reconstructions in oncologic and nontumoral conditions. Berlin: Springer Verlag, 1991:387-94.

5. Lee SH, Oh JH, Yoo KH, Kim HS. Infection after prosthetic reconstruction in limb salvage surgery. Int Orthop 2002;26:179-84.

6. Ward WG, Johnstone-Jones K, Lowenbraum S, et al. Antibiotic prophylaxis and infection resistance of massive tumor endoprostheses during chemotherapy. J South Orthop Assoc 1997;6:180-5.

7. Hillmann A, Hoffmann C, Gosheger G, Krakau H, Winkelmann W. Malignant tumor of the distal part of the femur or the proximal part of the tibia: endoprosthetic replacement or rotationplasty: functional outcome and quality-of-life measurements. J Bone Joint Surg [Am] 1999;81-A:462-7.

8. Lindner NJ, Ramm 0, Hillmann A, et al. Limb salvage and outcome of osteosarcoma: the university of Muenster experience. Clin Orthop 1999;358:83-9.

9. Gerrand CH, Currie D, Grigoris P, Reid R, Hamblen DL. Prosthetic reconstruction of the femur for primary bone sarcoma. Int Orthop 1999;23:286-90.

10. Ilyas I, Kurar A, Moreau PG, Younge DA. Modular megaprosthesis for distal femoral tumors. Int Orthop 2001;25:375-7.

11. Sanjay BK, Moreau PG. Limb salvage surgery in bone tumour with modular endoprosthesis. Int Orthop 1999;23:41-6.

12. Shih LY, Sim FH, Pritchard DJ, Rock MG, Chao EY. Segmental total knee arthroplasty after distal femoral resection for tumor. Clin Orthop 1993;292:269-81.

13. Enneking WF, Dunham W, Gebhardt MC, et al. A system for the functional evaulation of reconstructive procedures after surgical treatment of tumors of the musculoskeletal system. Clin Orthop 1993;286:241-6.

14. Mittermayer F, Krepler P, Dominkus M, et al. Long-term followup of uncemented tumor endoprostheses for the lower extremity. Clin Orthop 2001;388:167-77.

15. Horowitz SM, Glasser DB, Lane JM, Healey JH. Prosthetic and extremity survivorship after limb salvage for sarcoma: how long do the reconstructions last? Clin Orthop 1993;293:280-6.

16. Capanna R, Morris HG, Campanacci D, Del Ben M, Campanacci M. Modular uncemented prosthetic reconstruction after resection of tumours of the distal femur. J Bone Joint Surg [Br] 1994;76-B:178-86.

17. Plötz W, Rechl H, Burgkart R, et al. Limb salvage with tumor endoprostheses for malignant tumors of the knee. Clin Orthop 2002;405:207-15.

18. Kay RM, Kabo JM, Seeger LL, Eckardt JJ. Hydroxyapatite-coated distal femoral replacements: preliminary results. Clin Orthop 1994;302:92-100.

19. Unwin PS, Cannon SR, Grimer RJ, et al. Aseptic loosening in cemented custommade prosthetic replacements for bone tumours of the lower limb. J Bone Joint Surg [Br] 1996;78-B:5-13

20. Mascard E, Anract P, Touchene A, Pouillart P, Tomeno B. Complications from the hinged GUEPAR prosthesis after resection of knee tumor: 102 cases. Rev Chir Orthop Reparatrice Appar Mot 1998;84:628-37 (in French).

21. Bickels J, Wittig JC, Kollender Y, et al. Distal femur resection with endoprosthetic reconstruction: a long-term followup study. Clin Orthop 2002;400:225-35.

22. Kabo JM, Yang RS, Dorey FJ, Eckardt JJ. In vivo rotational stability of the kinematic rotating hinge knee prosthesis. Clin Orthop 1997;336:166-76.

23. Choong PFM, Sim FH, Pritchard DJ, Rock MG, Chao EY. Megaprostheses after resection of distal femoral tumors: a rotating hinge design in 30 patients followed for 2-7 years. Acta Orthop Scand 1996;67:345-51.

24. Kawai A, healey JH, Boland PJ, Athanasian EA, Jeon DG. A rotating-hinge knee replacement for malignant tumors of the femur and tibia. J Arthroplasty 1999; 14:187-96.

25. Grimer RJ, Carter SR, Tillman RM, et al. Endoprosthetic replacement of the proximal tibia. J Bone Joint Surg [Br] 1999;81-B:488-94.

26. Anract Ph, Missenard G, Jeanrot C, Dubois V, Tomeno B. Knee reconstruction with prosthesis and muscle flap after total arthrectomy. Clin Orthop 2001;384:208-16.

27. Petsching R, Baron R, Kotz R, RitschI P, Engel A. Muscle function after endoprosthetic replacement of the proximal tibia: different techniques for extensor reconstruction in 17 tumors patients. Acta Orthop Scand 1995;66:266-70 